- Original Article

\title{
Relationship between Dry Eye Syndrome and Frequency of Coffee Consumption in Korean Adults: Korea National Health and Nutrition Examination Survey V, 2010-2012
}

\author{
Kwon-Jin Jeong, Jong-Gi Choi, Eun-Joo Park, Hyo-Eun Kim, Sun-Mi Yoo, Seung-Guk Park* \\ Department of Family Medicine, Inje University Haeundae Paik Hospital, Busan, Korea
}

Background: Dry eye syndrome is a common health problem in the adult population. Many risk factors including age, sex, prior eye surgery, various chronic diseases, and lifestyle factors can affect its development. We have evaluated the risk of dry eye syndrome based on the frequency of coffee consumption among Korean adult population. Methods: A total of 9,752 adults with age 19 years and older were randomly selected between 2010 and 2012. They have all participated in the National Health and Nutrition Examination Survey V of Korea. Dry eye syndrome was being diagnosed by the physicians at some points in the participant's lifetime. The average daily coffee intake was divided into the following: less than 1 cup, 1 to 2 cups, and 3 cups or more. Various physio-environmental factors and medical conditions were used as correction variables to assess the risk of dry eye syndrome in relation to the frequency of coffee consumption.

Results: The prevalence of dry eye syndrome decreased to $9.2 \%, 8.8 \%$, and $6.3 \%$ as coffee consumption increased from less than 1 cup to 1-2 cups and more than 3 cups, respectively. However, there was no significant relationship between the frequency of coffee consumption and the risk of dry eye syndrome after adjusting various risk factors. Conclusion: There is no relationship between the frequency of coffee consumption and risk of dry eye syndrome.

Keywords: Dry Eye Syndromes; Coffee; Caffeine; Life Style

Received: March 22, 2017, Revised: June 30, 2017, Accepted: July 21, 2017

*Corresponding Author: Seung-Guk Park https://orcid.org/0000-0002-2986-3729

Tel: +82-51-797-3220, Fax: +82-51-797-0298, E-mail: sgpark@paik.ac.kr 


\section{INTRODUCTION}

Dry eye syndrome is a disease that causes various symptoms such as eye irritation, congestion, fatigue, and visual disturbances due to decreased tear production or excessive tear evaporation. ${ }^{1,2)}$ According to recent epidemiological studies, dry eye syndrome is a very common health problem in Asian countries with prevalence from $8.0 \%$ to $14.4 \% .^{3-5)}$ There is a wide range of risk factors that include age, sex, postmenopausal hormone therapy experience, presence of connective tissue disease, prior refractive surgery, and exposure to various medications such as antihistamines, antihypertensive, and antidepressant. ${ }^{6}$ Furthermore, a study in Korea has shown that residents in urban areas, people that work in offices, or those with medical conditions including hyperlipidemia, osteoarthritis, and renal failure are more at risk of dry eye syndrome. ${ }^{7)}$

In addition to medical reasons, various lifestyle factors can affect dry eye syndrome. For example, coffee intake have been thought to be a risk factor for dry eye syndrome due to the diuretic effect of caffeine, ${ }^{8)}$ but recent studies showed that caffeine is not associated with diuretic problems ${ }^{9)}$ and rather may reduce dry eye syndrome by increasing tear secretion in a Japanese experimental study. ${ }^{10)}$ However, only a few studies $^{11-13)}$ have been conducted on the relationship between coffee intake and dry eye syndrome, and previous studies did not give consistent results until now. Therefore, this study aims to investigate the risk of dry eye syndrome in relation to the frequency of coffee consumption based on the Korea National Health and Nutrition Examination Survey (KNHANES).

\section{METHODS}

\section{Study Population}

This study used data from the KNHANES V 2010-2012. Among the 19,599 participants aged 19 years and older, 9,752 were selected excluding those who have not had an eye examination and corresponding survey data. The KNHANES data is cross-sectional, nationally representative, and reliable in regard to the health status, health behavior, food, and nutrition status of Korean people. All participants provided written informed consent, and the study protocol was administered by the Korean Centers for Disease Control and Prevention (2010-02CON21-C, 2011-02COM-06-C, 2012-01EXP-01-2C).

\section{Definitions of Dry Eye Syndrome and Measurements}

The presence of dry eye syndrome was determined based on the answer "yes" to one of the survey questions from the health questionnaire, "Have you had been diagnosed with dry eye syndrome in the lifetime by physicians?"

The total intake of caffeine was calculated by about 67 to $74 \mathrm{mg}$ in a can of coffee or coffee mix. ${ }^{14)}$ The quantity of caffeine consumed when drinking "more than 3 cups" of coffee is about 201 to $222 \mathrm{mg}$ or more, which is equivalent to the capacity that can increase intraocular pressure according to some studies. ${ }^{15,16)}$ Based on this, the authors of this study divided the amount of coffee consumption by using three different subcategories. The frequency of coffee consumption was classified according to an answer from the various choices for "How often did you consume coffee in the last year?" Answers stating "rarely drink," "1-3 times a month," or "1-6 times a week" were defined as "averaging less than 1 cup per day." The average daily intake of 1 or more cups of coffee was divided into " $1-2$ cups per day" and " 3 cups or more per day."

Demographic variables included the level of education (middle or less, high school, university or more), type of occupation (indoor versus outdoor), and primary residential area (rural or urban). The residential area of a participant was considered urban if the city had a population of more than 1 million (Seoul, Busan, Daegu, Daejeon, Gwangju, Incheon, and Ulsan). All other residential areas were considered rural. The type of occupation was categorized as (1) managerial or other specialized jobs; (2) regular office job; (3) service or sales job; (4) job relating to agriculture, fishery, or forestry; (5) blue-collar job; (6) simple labor job; or just (7) unemployed. Choices 4, 5, and 6 were considered outdoor occupations. "Drinking" is defined as more than once a month of alcohol consumption and "non-drinking" as less than 1 drink per month. Smoking was categorized to (1) non-smokers or those who smoked less than 100 cigarettes in their lifetime and currently non-smoking, (2) past smokers or those who smoked more than 100 cigarettes in their lifetime and currently non-smoking, and (3) smokers or those who smoked more than 100 cigarettes in their lifetime and currently smoking. Physical activity was considered as 5 or more days of exercise per week with walks of 30 minutes or more.

Participants were considered to have a disease if they had a medical condition that included hypertension, hyperlipidemia, diabetes mellitus, chronic renal failure, degenerative arthritis, rheumatoid arthritis, thyroid disease, and depression. Hours of sleep was categorized as "less than 6 hours," " 6 to 8 hours," and " 9 hours or more" based on the results from a previous study. ${ }^{17}$

\section{Statistical Analyses}

According to the guidelines for the use of data of KNHANES V, variance estimation layer, stratification variables, and sample weights were used for the analysis. Continuous variables among the general characteristics of the participants were presented as mean and standard error by general linear regression analysis. Categorical variables were presented as frequency (percentage). A multivariate logistic regression analysis was performed for the risk of dry eye syndrome based on the average amount of coffee per day. We presented the adjusted odds ratios (ORs) and 95\% confidence intervals (CIs) after controlling for age, sex, body mass index (BMI), residential area, occupation, education level, physical activity, sleep, drinking, smoking, prior history of eye surgery, and medical conditions including hypertension, diabetes, osteoarthritis, thyroid disease, and depression. SAS ver. 9.2 (SAS Institute Inc., Cary, NC, USA) was used to perform all statistical analyses. P-values $<0.05$ were considered as statistically significant. 


\section{RESULTS}

\section{General Characteristics of Participants}

In 9,752 participants, the prevalence of dry eye syndrome was $8.4 \%$, among which $4.1 \%$ for men and $12.1 \%$ for women.

Table 1 shows the general characteristics of the three groups according to the amount of coffee consumption. Out of the total participants, 4,620 participants (47\%) consumed 1 to 2 cups of coffee a day, 3,384 participants (35\%) consumed less than 1 cup, and 1,748 participants $(18 \%)$ consumed 3 or more cups. The mean age of the participants was 45.46 years, and the mean age was the highest at 47.40 years in the participants with an average consumption of 1 to 2 cups of coffee.

Those who consumed more than 3 cups of coffee a day were men and current smokers and had higher BMI. They were also more likely to work outdoors, graduate high school or more, sleep 6 to 8 hours, and drink alcohol. There were fewer people with hypertension, diabetes, osteoarthritis, thyroid disease, and depression and history of ophthalmic surgery among them.

The prevalence of dry eye syndrome was higher among people who drink less than 1 cup of coffee per day (9.2\%) than those who drink 1-2 cups $(8.8 \%)$ or more than 3 cups of coffee per day (6.3\%).

\section{Relationship between Dry Eye Syndrome and the Frequency of Coffee Consumption}

Table 2 presents the risk of dry eye syndrome in relation to the amount of coffee consumption. Risk of dry eye syndrome was significantly low among people who drink 3 or more cups of coffee a day in model 1 adjusting for only age (OR, 0.661; 95\% CI, 0.497 to 0.879 ). However, this relationship was not significant after adjusting for sex in model 2 (OR, 0.836; $95 \% \mathrm{CI}, 0.626$ to 1.116 ). It was also not significant after further adjusting for BMI, residential area, working outdoors, education, physical activity, sleeping time, smoking, and alcohol intake in model 3 (OR, 0.908; 95\% CI, 0.654 to 1.260) or adjusting for history of chronic diseases and ophthalmic surgery in model 4 (OR, 0.969; 95\% CI, 0.693 to 1.353$)$.

\section{DISCUSSION}

This study evaluated the risk of dry eye syndrome according to the average consumption of coffee per day for Korean adults over 19 years of

Table 1. General characteristics according to the frequency of coffee consumption in Korean adults

\begin{tabular}{|c|c|c|c|c|}
\hline \multirow{2}{*}{ Characteristic } & \multicolumn{3}{|c|}{ Coffee consumption (cup/d) } & \multirow{2}{*}{ P-value } \\
\hline & $<1(n=3,384)$ & $1-2(n=4,620)$ & $\geq 3(n=1,748)$ & \\
\hline Age (y) & $43.92 \pm 0.50$ & $47.40 \pm 0.52$ & $45.05 \pm 0.54$ & $<0.001$ \\
\hline Men (\%) & $41.3(1.1)$ & $41.8(0.8)$ & $63.6(1.3)$ & $<0.001$ \\
\hline Body mass index $\left(\mathrm{kg} / \mathrm{m}^{2}\right)$ & $23.22 \pm 0.08$ & $23.77 \pm 0.96$ & $24.04 \pm 0.13$ & $<0.001$ \\
\hline Rural residence (\%) & $55.0(1.7)$ & $51.8(1.6)$ & $55.5(2.0)$ & 0.039 \\
\hline Working outdoors (\%) & $22.2(1.2)$ & $27.7(1.2)$ & $35.3(1.7)$ & $<0.001$ \\
\hline Education (\%) & & & & $<0.001$ \\
\hline Middle school or less & $31.8(1.2)$ & $32.2(1.2)$ & $23.5(1.4)$ & \\
\hline High school & $37.8(1.2)$ & $35.3(1.1)$ & $40.7(1.4)$ & \\
\hline University or more & $30.4(1.1)$ & $32.5(1.1)$ & $35.8(1.5)$ & \\
\hline Physically active (\%) & $17.8(0.9)$ & $16.4(0.7)$ & $14.1(1.0)$ & 0.036 \\
\hline Duration of sleep time (h/d) (\%) & & & & $<0.001$ \\
\hline Less than 6 & $10.0(0.7)$ & $6.9(0.5)$ & $5.7(0.7)$ & \\
\hline $6-8$ & $75.1(0.9)$ & $80.2(0.8)$ & $81.7(1.3)$ & \\
\hline 9 or more & $14.9(0.7)$ & $12.8(0.7)$ & $12.6(1.0)$ & \\
\hline Alcohol drinking (\%) & $60.2(1.2)$ & $63.3(1.1)$ & $72.4(1.4)$ & $<0.001$ \\
\hline Smoking (\%) & & & & $<0.001$ \\
\hline Never & $66.8(1.1)$ & $61.9(0.8)$ & $37.0(1.3)$ & \\
\hline Past & $15.8(0.8)$ & $18.5(0.7)$ & $18.5(1.1)$ & \\
\hline Current & $17.4(0.9)$ & $19.6(0.8)$ & $44.5(1.4)$ & \\
\hline History of ophthalmic surgery (\%) & $12.6(0.7)$ & $11.9(0.6)$ & $8.1(0.7)$ & $<0.001$ \\
\hline Hypertension (\%) & $17.3(0.8)$ & $18.8(0.7)$ & $14.3(1.1)$ & 0.001 \\
\hline Diabetes (\%) & $7.7(0.6)$ & $7.0(0.4)$ & $4.0(0.5)$ & $<0.001$ \\
\hline Hyperlipidemia (\%) & $8.1(0.6)$ & $8.6(0.5)$ & $7.5(0.8)$ & 0.414 \\
\hline Osteoarthritis (\%) & $9.7(0.5)$ & $9.8(0.5)$ & $4.6(0.6)$ & $<0.001$ \\
\hline Rheumatoid arthritis (\%) & $1.9(0.2)$ & $1.7(0.2)$ & $1.5(0.3)$ & 0.688 \\
\hline Thyroid disease (\%) & $3.9(0.4)$ & $3.8(0.3)$ & $2.2(0.4)$ & 0.01 \\
\hline Renal disease (\%) & $0.4(0.1)$ & $0.3(0.1)$ & $0.1(0.1)$ & 0.329 \\
\hline Depression (\%) & $4.7(0.5)$ & $3.6(0.3)$ & $2.5(0.5)$ & 0.004 \\
\hline Dry eye syndrome (\%) & $9.2(0.7)$ & $8.8(0.5)$ & $6.3(0.7)$ & 0.006 \\
\hline
\end{tabular}

Values are presented as mean \pm SE or \% (SE).

$\mathrm{SE}$, standard error.

*By analysis of variance or chi-square test. 
Table 2. Risk of dry eye syndrome according to the frequency of coffee consumption in Korean adults

\begin{tabular}{cccc}
\hline \multirow{2}{*}{ Variable } & \multicolumn{3}{c}{ Coffee consumption (cup/d) } \\
\cline { 2 - 4 } & $<1$ & $1-2$ & $\geq 3$ \\
\hline${\text { Model } 1^{*}}^{*}$ & 1.000 & $0.957(0.794-1.153)$ & $0.661(0.497-0.879)$ \\
Model 2 $^{\dagger}$ & 1.000 & $0.962(0.800-1.157)$ & $0.836(0.626-1.116)$ \\
Model $^{\ddagger}$ & 1.000 & $1.019(0.830-1.251)$ & $0.908(0.654-1.260)$ \\
Model $^{\S}$ & 1.000 & $1.047(0.851-1.290)$ & $0.969(0.693-1.353)$ \\
\hline
\end{tabular}

Values are presented as odds ratio (95\% confidence interval). Multivariable logistic regression analysis was used.

${ }^{*}$ Adjusted for age. ${ }^{\dagger}$ Adjusted for age and sex. ${ }^{\ddagger}$ Adjusted for age, sex, body mass index, residential area, occupation, education, physical activity, sleep time, smoking, and alcohol intake. ${ }^{\S}$ Adjusted for age, sex, body mass index, residential area, occupation, education, physical activity, sleep time, smoking, alcohol intake, ophthalmic surgery, diabetes, hypertension, osteoarthritis, thyroid disease, and depression.

age from the data of KNHANES V. Prevalence of dry eye syndrome was lower in the group that consumed more than 3 cups of coffee than in the group drinking less than 1 cup, but the risk of dry eye syndrome was not related to the frequency of coffee consumption after adjusting for risk factors including sex, lifestyle, and chronic disease.

The result corresponds to the earlier studies especially the one published by Chia et al. ${ }^{11)}$ in Australia of 3,654 people over the age of 50 years. They attempted to compare the people that consume at least 1 cup of caffeinated beverage a day to those who did not but had reached the conclusion that there were no differences that had significant effect on dry eye syndrome. Moss et al. ${ }^{12)}$ had tried tracing the cohort of 2,414 people over the age of 43 years for 10 years to find out whether drinking caffeine would increase the risk of dry eye syndrome. The study showed that the incidence of dry eye syndrome in caffeine drinkers and non-drinkers was similar (21.2\% versus $23.2 \%, \mathrm{P}=0.39$ ), and caffeine intake was not a significant risk factor for dry eye syndrome.

The same researchers had reported in an earlier cross-sectional study that the prevalence of dry eye syndrome was significantly different in caffeine consumers and non-consumers (13\% versus $16.6 \%$, $\mathrm{P}<0.001),{ }^{13)}$ but the cohort study to confirm causality did not find this association.

In our study, the prevalence of dry eye syndrome in people who had more than 3 cups of coffee $(6.3 \%)$ was lower than that of those who had less than 1 cup a day (9.2\%). It may be related that there were less women, more young people, and fewer people with chronic diseases in the group that had consumed 3 or more cups of coffee. It is well known that the risk of dry eye syndrome is higher in women, people with chronic diseases, and older individuals. ${ }^{7,18-21)}$ The cross-sectional study of Moss et al. ${ }^{13)}$ also showed that men and younger people had lower risk of dry eye syndrome. Because our study only selected those who were diagnosed by the physician to be having dry eye syndrome, people whose symptoms were mild would not have been included. In a previous study analyzing KNHANES data, the prevalence of the dry eye syndrome was $8.0 \%$ on the basis of the physician's diagnosis and
$14.4 \%$ based on symptoms. ${ }^{3)}$

Sex is an important risk factor for dry eye syndrome. In our study, there was a sex difference between those who consumed more than 3 cups of coffee and those who consume less, and multivariable analysis revealed that the risk of dry eye syndrome became insignificant after adjusting for sex. When we attempted the secondary analysis after separating participants by sex, we again found that the frequency of coffee consumption had no relationship with dry eye syndrome (data are not presented).

The limitation of our study is that, first, we might not include people with mild symptoms because we selected only those who were diagnosed by physicians with dry eye syndrome. There is also a possibility that the diagnosis was not accurate because the self-reported questionnaire was used without ophthalmic examinations. Second, this research is a cross-sectional study that cannot define causality between coffee intake and dry eye syndrome. Finally, it is not only coffee that contains caffeine as its ingredient, but we did not take into account consumption of other foods and drinks with caffeine. However, coffee is the major source of caffeine in adults, and the strength of our study was precisely quantifying the amount of coffee intake. In addition, previous studies focused on a small group of young or old aged people, ${ }^{10-13)}$ but our study had a broad range of age groups from young to old. It was also a great advantage of this research to use data from a nationally representative sample.

In conclusion, we have found that there was no significant relationship between dry eye syndrome and the frequency of coffee consumption in Korean adults. In the future, it will be necessary to clarify the causal relationship through various longitudinal or experimental studies on the risk of dry eye syndrome according to the frequency of coffee consumption.

\section{CONFLICT OF INTEREST}

No potential conflict of interest relevant to this article was reported.

\section{REFERENCES}

1. Lin PY, Tsai SY, Cheng CY, Liu JH, Chou P, Hsu WM. Prevalence of dry eye among an elderly Chinese population in Taiwan: the Shihpai Eye Study. Ophthalmology 2003;110:1096-101.

2. McCarty CA, Bansal AK, Livingston PM, Stanislavsky YL, Taylor HR. The epidemiology of dry eye in Melbourne, Australia. Ophthalmology 1998;105:1114-9.

3. Ahn JM, Lee SH, Rim TH, Park RJ, Yang HS, Kim TI, et al. Prevalence of and risk factors associated with dry eye: the Korea National Health and Nutrition Examination Survey 2010-2011. Am J Ophthalmol 2014;158:1205-14.

4. Tan LL, Morgan P, Cai ZQ, Straughan RA. Prevalence of and risk factors for symptomatic dry eye disease in Singapore. Clin Exp Optom 2015;98:45-53.

5. Li J, Zheng K, Deng Z, Zheng J, Ma H, Sun L, et al. Prevalence and risk factors of dry eye disease among a hospital-based population in 
southeast China. Eye Contact Lens 2015;41:44-50.

6. American Academy of Ophthalmology, Cornea/External Disease Panel. Preferred practice pattern guidelines: dry eye syndrome [Internet]. San Francisco (CA): American Academy of Ophthalmology; 2013 [cited 2013 Sep 21]. Available from: http://www.aao.org/ppp.

7. Roh HC, Lee JK, Kim M, Oh JH, Chang MW, Chuck RS, et al. Systemic comorbidities of dry eye syndrome: the Korean National Health and Nutrition Examination Survey V, 2010 to 2012. Cornea 2016;35:187-92.

8. Geiger M. Hold the caffeine, pass the fish. Rev Optom [Internet]. 2003 [cited 2003 Feb 18];140:[about 7 screens]. Available from: https:// www.reviewofoptometry.com.

9. Neuhäuser-Berthold, Beine S, Verwied SC, Luhrmann PM. Coffee consumption and total body water homeostasis as measured by fluid balance and bioelectrical impedance analysis. Ann Nutr Metab 1997;41:29-36.

10. Osei KA, Ovenseri-Ogbomo G, Kyei S, Ntodie M. The effect of caffeine on tear secretion. Optom Vis Sci 2014;91:171-7.

11. Chia EM, Mitchell P, Rochtchina E, Lee AJ, Maroun R, Wang JJ. Prevalence and associations of dry eye syndrome in an older population: the Blue Mountains Eye Study. Clin Exp Ophthalmol 2003;31:229-32.

12. Moss SE, Klein R, Klein BE. Long-term incidence of dry eye in an older population. Optom Vis Sci 2008;85:668-74.
13. Moss SE, Klein R, Klein BE. Prevalence of and risk factors for dry eye syndrome. Arch Ophthalmol 2000;118:1264-8.

14. Korea Ministry of Food and Drug Safety. Korea food additives code. Seoul: Korea Food Industry Association; 2015.

15. Avisar R, Avisar E, Weinberger D. Effect of coffee consumption on intraocular pressure. Ann Pharmacother 2002;36:992-5.

16. Chandrasekaran S, Rochtchina E, Mitchell P. Effects of caffeine on intraocular pressure: the Blue Mountains Eye Study. J Glaucoma 2005;14:504-7.

17. Lee W, Lim SS, Won JU, Roh J, Lee JH, Seok H, et al. The association between sleep duration and dry eye syndrome among Korean adults. Sleep Med 2015;16:1327-31.

18. Kastelan S, Tomic M, Salopek-Rabatic J, Novak B. Diagnostic procedures and management of dry eye. Biomed Res Int 2013;2013:309723.

19. Gayton JL. Etiology, prevalence, and treatment of dry eye disease. Clin Ophthalmol 2009;3:405-12.

20. Tang YL, Cheng YL, Ren YP, Yu XN, Shentu XC. Metabolic syndrome risk factors and dry eye syndrome: a meta-analysis. Int J Ophthalmol 2016;9:1038-45.

21. Dao AH, Spindle JD, Harp BA, Jacob A, Chuang AZ, Yee RW. Association of dyslipidemia in moderate to severe meibomian gland dysfunction. Am J Ophthalmol 2010;150:371-5. 